\title{
High-concentration Epigallocatechin Gallate Treatment Causes Endoplasmic Reticulum Stress-mediated Cell Death in HepG2 Cells
}

\author{
Joon-lk Ahn, Kyoung Ji Jeong, Moon-Jeong \\ Ko, Hee Jung Shin, Hye Joo Chung* and \\ Ho-Sang Jeong*
}

Molecular Pharmacology Division, Pharmacological Research Department, National Institute of Toxicological Research, Seoul 122-704, Korea

\begin{abstract}
Epigallocatechin gallate (EGCG), a well-known antioxidant molecule, has been reported to cause hepatotoxicity when used in excess. However, the mechanism underlying EGCG-induced hepatotoxicity is still unclear. To better understand the mode of action of EGCG-induced hepatotoxicity, we examined the effect of EGCG on human hepatic gene expression in HepG2 cells using microarrays. Analyses of microarray data revealed more than 1300 differentially expressed genes with a variety of biological processes. Upregulated genes showed a primary involvement with protein-related biological processes, such as protein synthesis, protein modification, and protein trafficking, while downregulated genes demonstrated a strong association with lipid transport. Genes involved in cellular stress responses were highly upregulated by EGCG treatment, in particular genes involved in endoplasmic reticulum (ER) stress, such as GADD153, GADD34, and ATF3. In addition, changes in genes responsible for cholesterol synthesis and lipid transport were also observed, which explains the high accumulation of EGCG-induced lipids. We also identified other regulatory genes that might aid in clarifying the molecular mechanism underlying EGCG-induced hepatotoxicity.
\end{abstract}

Keywords: endoplasmic reticulum stress, epigallocatechin gallate, hepatotoxicity, microarray

\section{Introduction}

Green tea is a traditional Asian beverage made from the

\footnotetext{
${ }^{*}$ Corresponding author: E-mail hosa33@kfda.go.kr hjchung@kfda.go.kr
}

Tel +82-2-380-1804, Fax +82-2-388-6457

Accepted 4 June 2009 leaves of Camellia sinensis and contains several polyphenolic components, such as catechin, epicatechin, epicatechin gallate (ECG), epigallocatechin (EGC), and epigallocatechin gallate (EGCG).

In addition to its prominent role as a common beverage, green tea has demonstrated profound biochemical and pharmacological activities, including antioxidative activities and anti-inflammatory and anticarcinogenic properties (Isbrucker et al., 2006). The beneficial effects of green tea on health have been backed by multiple scientific reports, which have proposed the involvement of green tea in various biological processes, such as inhibition of: telomerase, mitogen-activated protein kinase (MAPK), activator protein-1, nuclear factor (NF)-kB, binding of epidermal growth factor to its receptor, angiogenesis, and activation of apoptosis (Vittal et al., 2004).

However, it is becoming more apparent that green tea compounds might also cause deleterious effects at pharmacological concentrations in certain cases (Lambert et al., 2007). For example, in France and Spain, cases of hepatic attack have been reported after the use of hydroalcoholic extracts of green tea as a complement of diets. These observations have led to the suspension of marketing authorization of these products in both countries. In animal models, EGCG causes a high mortality rate in adult female Swiss Webster mice and hepatotoxicity in immature C57BL/6 female mice (Galati et al., 2006; Goodin and Rosengren, 2003). Additionally, recent studies have shown that several phenolic antioxidant food additives can accelerate oxidative damage to DNA, proteins, and carbohydrates, despite their antioxidative action. The molecular mechanism of prooxidative action and hepatotoxicity that are induced by EGCG remains poorly understood. Galati et al. (2006) reported that cell death induced by EGCG was associated with increased production of reactive oxygen species (ROS) and depletion of reduced glutathione (GSH) and catechol-o-methyltransferase (COMT). The mechanism of EGCG-induced cell death was suggested in previous reports, which implicated the apoptosis pathway (Schmidt et al. 2005) and the endoplasmic reticulum (ER) stress-related pathway (Dodo et al., 2008).

To gain insight into the molecular mechanism underlying EGCG-induced cytotoxicity, we used a microarray approach, which allowed us to observe the global effects of EGCG on hepatic gene expression. Time course 
observations of the gene expression profile were made. We propose in the present study that hepatic cell death resulting from high-concentration EGCG treatment is mainly caused by ER stress.

\section{Methods}

\section{Chemicals}

EGCG was purchased from Sigma (St. Louis, MO, USA). DMEM, fetal bovine serum (FBS), penicillin/streptomycin, TRIzol, and DEPC-treated water were obtained from Invitrogen (Carlsbad, CA, USA).

\section{Cell culture}

The HepG2 cell line was cultured in DMEM medium supplemented with $10 \%$ heat-inactivated FBS, $100 \mathrm{U} / \mathrm{ml}$ penicillin, and $100 \mathrm{mg} / \mathrm{ml}$ streptomycin and maintained in a monolayer culture at $37^{\circ} \mathrm{C}$ in an incubator of humidified air with $5 \% \mathrm{CO}_{2}$. Subconfluent cells $(80 \%)$ were passaged with a solution containing $0.05 \%$ trypsin and $0.02 \%$ EDTA.

\section{Cell viability assay}

HepG2 cells were treated with test substances dissolved in $10 \%$ DMSO for $24 \mathrm{~h}$. After the treatment, cell viability was assessed using the resazurin assay (CellTiter Blue ${ }^{\mathbb{R}}$, Promega, Madison, WI, USA). Cells were incubated with medium containing resazurin. A kinetic assay of resorufin, produced from resazurin by vital cells, was performed by measuring its absorbance $(570 \mathrm{~nm})$ in a SpectraMax microplate reader (Molecular Devices, Sunnyvale, CA, USA). The absorbance at $600 \mathrm{~nm}$ was used as reference.

\section{RNA isolation and DNA microarray}

Total RNA was extracted using Trizol at each time point in accordance with the manufacturer's procedures. RNA quantification and purity (260/280 ratio) were determined with a UV/VIS spectrophotometer (ND-1000, NanoDrop Technologies, Wilmington, DE, USA).

The Applied Biosystems Human Genome Survey Arrays (Applied Biosystems, Foster City, CA, USA), which contain 60-mer oligonucleotide probes representing a set of 32,878 individual mouse genes and more than 250 control probes, were used for differential gene expression profiles. Microarray experiments were performed according to the manufacturer's instructions. Digoxigenin (DIG)-UTP-labeled CRNA was generated from $5 \mu \mathrm{g}$ of total RNA and amplified using a chem- iluminescent reverse transcription (RT) in vitro transcription labeling kit (Applied Biosystems). Briefly, each microarray was prehybridized in hybridization buffer with blocking reagent at $55^{\circ} \mathrm{C}$ for $1 \mathrm{~h}$. DIG-labeled cRNA targets $(10 \mu \mathrm{g})$ were fragmented to $100 ? 400 \mathrm{bp}$ and hybridized with each prehybridized microarray at $55^{\circ} \mathrm{C}$ for 16 $h$. The arrays were washed with hybridization wash buffer and then with chemiluminescence rinse buffer. Chemiluminescent signals were generated by incubating the arrays with anti-DIG-alkaline phosphatase and chemiluminescence substrate. Images were collected for each microarray using the 1700 Chemiluminescent Microarray Analyzer (Applied Biosystems). Microarray images were autogridded, and the chemiluminescent signals were quantified, corrected for background, spatially normalized, and exported for a quality report. Microarray data with quality reports above the manufacturer's threshold were used for further analysis.

\section{Analysis of microarray expression data}

Signal intensities were imported into GenPlex software (Istech Inc, Korea), where interarray quantile normalization was performed in order to minimize the effect of external variables introduced into the data. Quality filtering of unreliable spots (flag value $<100$ and $\mathrm{S} / \mathrm{N}<3$ ) was performed before normalization. Then, the expression intensities were log2-transformed. Differentially expressed genes (DEGs) were selected by one-way ANOVA, and correction for multiple testing was then performed using Benjamini-Hochberg multiple testing to derive a false discovery rate estimate from the raw p-values. A false discovery rate of $1 \%$ was used as a cutoff for statistical significance. We took the average value from the gene expression ratio obtained in 3 or more biological replicates.

For further analysis, DEGs were divided into gene clusters according to expression level at each time point. Hierarchical clustering was applied to genes using Euclidean distance as a similarity measurement, as implemented in the Genesis software program (http:// genome.tugraz.at). Additionally, DEGs were divided into categories according to biological process using the PANTHER (Protein ANalysis THrough Evolutionary Relationships) Protein Classification System (Applied Biosystems, https://panther.appliedbiosystems.com).

\section{Semiquantitative and real-time quantitative RT-PCR}

For semiquantitative and real-time quantitative RT-PCR, glyceraldehydes-3-phosphate dehydrogenase (GAPDH) was used as an internal control with the 5'- ccatcatgaagtgtgacgttg $-3^{\prime}$ and 5'- gtgctaggagccagagcagta -3 ' 
primer set. For each sample, the same total RNA used for microarray hybridization was reverse-transcribed using $1 \mu \mathrm{M}$ oligo (dT) primer with Superscript III reverse transcriptase (Invitrogen). The thermal profiles consisted of $94^{\circ} \mathrm{C}$ for $5 \mathrm{~min}$ for initial denaturing, followed by $25 \sim$ 35 cycles of $94^{\circ} \mathrm{C}$ for $30 \mathrm{~s}, 55 \sim 65^{\circ} \mathrm{C}$ for $30 \mathrm{~s}$, and $72^{\circ} \mathrm{C}$ for $30 \mathrm{~s}$ to $1 \mathrm{~min}$. All RT-PCR reactions were repeated at least 3 times. Triplicate real-time RT-PCR experiments were performed using the MiniOpticon system (Bio-Rad, Hercules, CA) and the DyNAmo Flash SYBR Green qPCR kit (Finnzymes, Espoo, Finland). A melting curve was obtained for each PCR product after each run to confirm that the signal corresponded to a unique amplicon of the product size. The primers were 5'- gaa gtg agt gct tct gcc atc -3 ' and 5'- act ttc cag ctt ctc cga ctc-3' for activating transcription factor 3 (ATF3); 5'- ttt cca gac tga tcc aac tgc -3' and 5'- acc act ctg ttt ccg ttt cct g -3' for DNA damage-inducible transcript 3 (DDIT3/GADD153/CHOP); 5'- gat gaa gag gag ggt gag gtc -3 ' and 5'- aca cct gta gca gga gtg gaa $\mathrm{g}-3$ ' for protein phosphatase, regulatory subunit 15A (PPP1R15A/GADD34); 5'- cca tac gga gaa acc aga aca -3 ' and 5'- ggt ggc ctt cgt act tct ctt g -3 ' for seven in absentia homolog 2 (SIAH2); 5'- gct ggt acc cag ctc ctc tac -3' and 5'- gtt ctc cag cac cag ctt ctt c -3' for tribbles homolog 3 (TRIB3); 5'- tca gtc acg ggt gag cag tat $g-3$ ' and 5'- caa agt ctc tgc caa gag tgg -3 ' for stromal cell-derived factor 2-like 1 (SDF2L1); 5' - cct tgt tcc aga gca gat gtc -3 ' and 5'- att cca cca aaa act gca cac -3' for choroideremia-like (CHML); and 5'- tca gct ttt tcc aga gat cca g $-3^{\prime}$ and 5'- cta tcc tgt cga ctt gct tgg -3' for YKT6 v-SNARE homolog (YKT6).

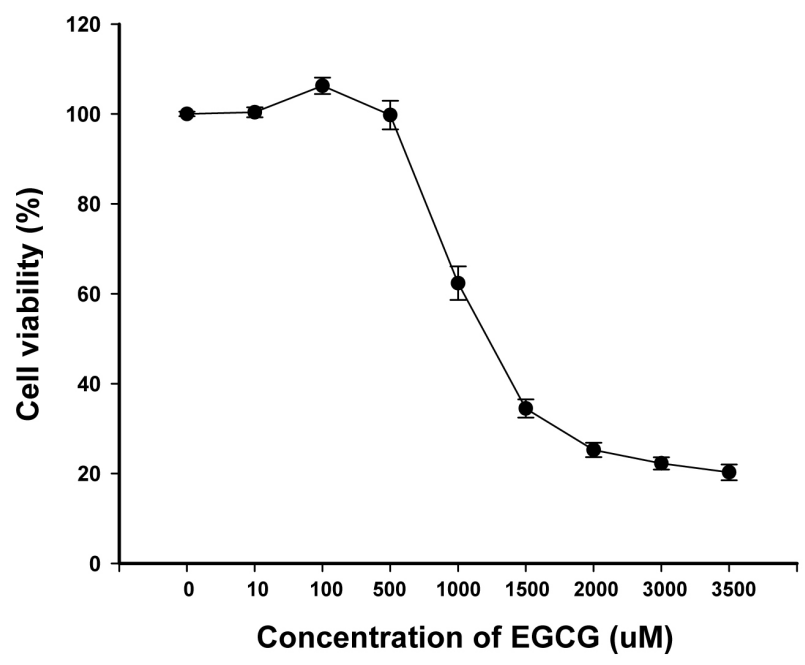

Fig. 1. Resazurin assay results from HepG2 cells treated with EGCG. Cells were incubated with the indicated concentrations of EGCG for $24 \mathrm{hr}$, followed by Resazurin assay. Each concentration was assayed in triplicate within an experiment. Data are expressed as percentage of untreated cells, and error bars represent the standard error of the mean.
A

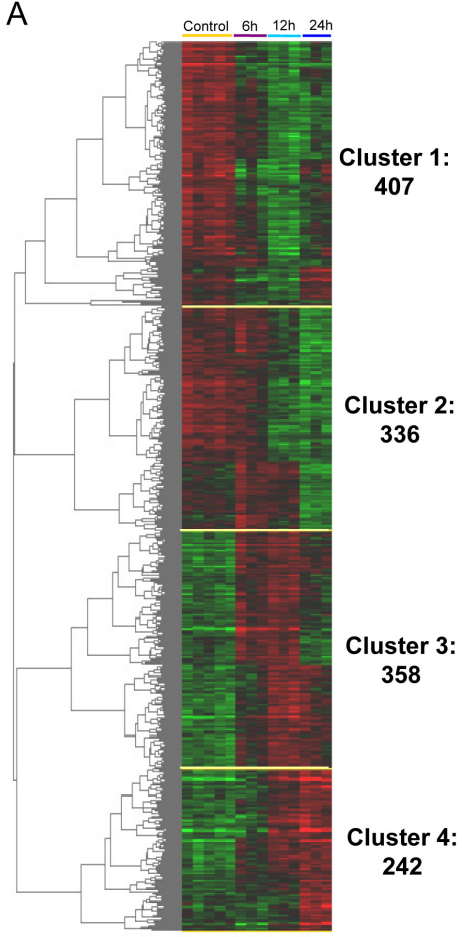

B

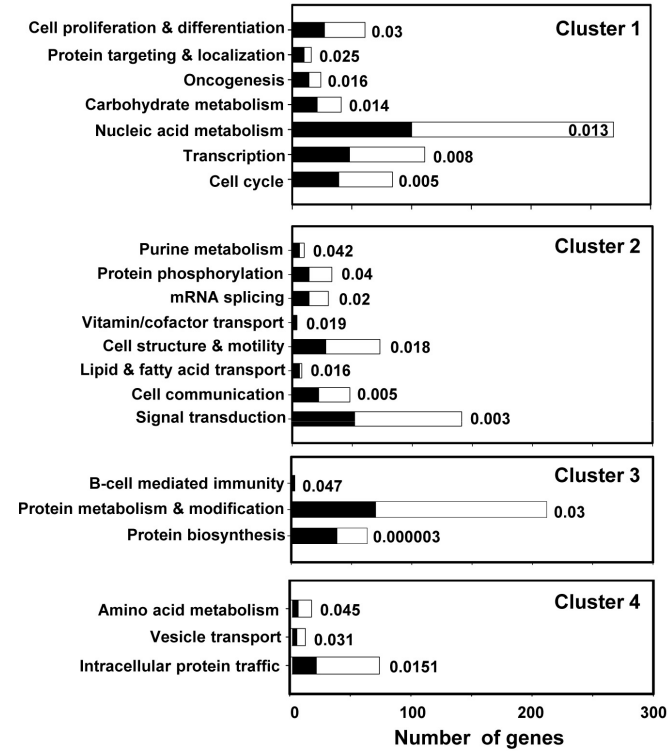

Fig. 2. (A) Hierarchical clustering of 1343 genes that showed differential expression patterns. The genes were clustered by Euclidean distance; 1343 genes were classified into 4 clusters. The intensity of red and green is proportional to the relative gene induction (red) or repression (green). (B) Primary biological processes for each cluster. The PANTHER gene expression data analysis tool was used to identify significantly overrepresented biological processes for each cluster. The bar graph indicates the numbers of genes belonging to specific biological processes. The $p$ value for each biological process is indicated. 


\section{Statistical analysis}

All experiments were carried out 3 times. Results are presented as mean values $\pm \mathrm{SE}$.

\section{Results}

\section{Cell viability following treatment with EGCG}

The effect of EGCG on HepG2 viability was monitored by resazurin assay, which measures the reducing capacity of mitochondria in cells. Based on the cell viability assay results, it seemed that the treatment of EGCG at low concentrations (10-100 uM) increased the viability of the cells but not at higher concentrations (1000-3500 uM). At higher concentrations, rather, EGCG caused a significant decrease in cell viability (Fig. 1). The lethal concentration $30\left(\mathrm{LC}_{30}\right)$ of EGCG against HepG2 cells was determined as $850 \mathrm{uM}$, according to nonlinear regression analysis. EGCC at $850 \mathrm{uM}$ was used for the following experiments.

\section{Identification of differentially expressed genes}

Gene expression profiling was performed to examine the temporal changes of gene expression in EGCG-treated HepG2 cells. Total RNA was prepared from the cells treated with $850 \mathrm{uM}$ of EGCG for $6,12,24 \mathrm{~h}$, and $0 \mathrm{~h}$

Table 1. Protein biosynthesis- and protein metabolism/modification-related genes regulated by EGCG

\begin{tabular}{|c|c|c|c|c|c|}
\hline Probe id & Gene symbol & Gene name & $\begin{array}{c}\text { 6h ratio } \\
-\log 2\end{array}$ & $\begin{array}{c}12 \mathrm{~h} \text { ratio } \\
-\log 2\end{array}$ & $\begin{array}{c}24 \mathrm{~h} \text { ratio } \\
-\log 2\end{array}$ \\
\hline \multicolumn{6}{|c|}{ Protein biosynthesis } \\
\hline 120032 & $\mathrm{EIF} 2 \mathrm{C} 2$ & eukaryotic translation initiation factor $2 \mathrm{C}, 2$ & 0.897 & 0.778 & 0.831 \\
\hline 203208 & EIF3S12 & eukaryotic translation initiation factor 3 , subunit 12 & 0.604 & 0.826 & 0.394 \\
\hline 185066 & ITGB4BP & integrin beta 4 binding protein & 1.210 & 1.495 & 0.733 \\
\hline 124345 & LOC134505 & similar to eukaryotic translation initiation factor 3 subunit $k$ & 0.851 & 1.265 & 0.508 \\
\hline 132913 & MRPL17 & mitochondrial ribosomal protein L17 & 0.773 & 0.977 & 0.394 \\
\hline 165531 & MRPL21 & mitochondrial ribosomal protein L21 & 0.781 & 1.310 & 0.601 \\
\hline 105643 & MRPL40 & mitochondrial ribosomal protein $\mathrm{L} 40$ & 0.653 & 0.722 & 0.700 \\
\hline 149811 & MRPL49 & mitochondrial ribosomal protein L49 & 0.580 & 1.299 & 0.973 \\
\hline 167428 & RPL28 & ribosomal protein L28 & 0.769 & 0.979 & 0.757 \\
\hline 184007 & RPS19 & ribosomal protein S19 & 0.379 & 1.038 & 0.739 \\
\hline 141447 & RPS9 & ribosomal protein S9 & 0.579 & 1.134 & 0.862 \\
\hline \multicolumn{6}{|c|}{ Protein metabolism \& modification } \\
\hline 208619 & BAG4 & BCL2-associated athanogene 4 & 1.697 & 1.673 & 1.389 \\
\hline 171701 & DNAJB6 & DnaJ (Hsp40) homolog, subfamily B, member 6 & 1.317 & 1.326 & 0.356 \\
\hline 140616 & DUSP23 & dual specificity phosphatase 23 & 0.982 & 1.245 & 0.550 \\
\hline 121612 & DUSP5 & dual specificity phosphatase 5 & 2.526 & 1.996 & 0.794 \\
\hline 151197 & F12 & coagulation factor XII (Hageman factor) & 0.830 & 1.115 & 0.971 \\
\hline 165933 & FLJ22405 & hypothetical protein FLJ22405 & 0.997 & 0.958 & 0.277 \\
\hline 111748 & HTRA1 & HtrA serine peptidase 1 & 0.724 & 0.854 & -0.460 \\
\hline 131931 & ICAM3 & intercellular adhesion molecule 3 & 1.046 & 1.715 & 1.360 \\
\hline 163269 & IMMP2L & IMP2 inner mitochondrial membrane peptidase-like (S. cerevisiae) & 0.887 & 1.461 & 0.380 \\
\hline 147822 & LATS2 & LATS, large tumor suppressor, homolog 2 (Drosophila) & 0.882 & 1.758 & 0.177 \\
\hline 135861 & MGC11102 & hypothetical protein MGC11102 & 0.793 & 1.643 & 1.231 \\
\hline 182792 & MSI2 & musashi homolog 2 (Drosophila) & 1.161 & 1.857 & 1.196 \\
\hline 165933 & MTMR14 & myotubularin related protein 14 & 0.997 & 0.958 & 0.277 \\
\hline 204476 & PPIH & peptidylprolyl isomerase $\mathrm{H}$ (cyclophilin $\mathrm{H}$ ) & 0.822 & 0.632 & 0.224 \\
\hline 184060 & PPP2R4 & protein phosphatase $2 A$, regulatory subunit $B^{\prime}$ (PR 53) & 0.711 & 0.859 & 0.451 \\
\hline 139782 & PRSS21 & protease, serine, 21 (testisin) & 0.897 & 0.758 & -0.091 \\
\hline 135806 & RNF113A & ring finger protein $113 \mathrm{~A}$ & 1.069 & 1.250 & 0.691 \\
\hline 163408 & $\mathrm{SIAH} 2$ & seven in absentia homolog 2 (Drosophila) & 2.187 & 2.766 & 1.249 \\
\hline 151579 & ST3GAL1 & ST3 beta-galactoside alpha-2,3-sialyltransferase 1 & 0.609 & 0.769 & -0.749 \\
\hline 113737 & TRIB3 & tribbles homolog 3 (Drosophila) & 1.597 & 2.620 & 2.246 \\
\hline 197340 & UBC & ubiquitin $\mathrm{C}$ & 0.768 & 0.925 & 0.321 \\
\hline 186200 & UBE2L3 & ubiquitin-conjugating enzyme E2L 3 & 0.722 & 0.843 & 0.399 \\
\hline 197127 & WARS & tryptophanyl-tRNA synthetase & 0.918 & 1.291 & 1.331 \\
\hline
\end{tabular}

\footnotetext{
${ }^{\dagger}$ Unknown genes removed.
} 
(the control). The cRNAs were subsequently synthesized and hybridized. Analyses revealed that EGCG treatment significantly $(p<0.01)$ altered the expression of 1343 genes (http://kotis.nifds.go.kr). Interestingly, activating transcription factor 3 (ATF3) and N-myc downstream-regulated gene 1 (NDRG1) showed marked overexpression (more than 5-fold), while inhibitor of DNA binding 1 (ID1) revealed a drastic downregulation (more than 10-fold) by EGCG treatment at all 3 time points.

\section{Clustering and functional enrichment analysis}

To characterize patterns of transcript expression during the time course, hierarchical clustering was performed on the 1343 genes, and these genes were classified into 4 clusters (Fig. 2A). The temporal cluster analysis revealed 4 clusters of transcripts that were either downregulated (cluster 1; 407 genes and cluster 2; 336 genes) or upregulated (cluster 3; 358 genes and cluster 4; 242 genes). Genes in cluster 1 showed a trend of expression that decreased up to $12 \mathrm{~h}$ and increased slightly at $24 \mathrm{~h}$. Genes in cluster 2 showed a gradual decrease up to $24 \mathrm{~h}$. On the contrary, genes in cluster 3 showed overexpression up to $12 \mathrm{~h}$ and slight underexpression at $24 \mathrm{~h}$. Cluster 4 contained genes with gradually increasing expression up to $24 \mathrm{~h}$.
To understand the effect of EGCG treatment on HepG2 cells, overrepresented biological processes of each cluster were identified using the PANTHER gene expression data analysis tool (Fig. 2B). According to the analyses, biological processes, such as cell cycle, transcription, nucleic acid metabolism, carbohydrate metabolism, oncogenesis, protein targeting/localization, and cell proliferation/differentiation, showed significant enrichment in cluster 1 , and signal transduction, cell communication, lipid/fatty acid transport, cell structure and motility, vitamin/cofactor transport, mRNA splicing, protein phosphorylation, and purine metabolism were in cluster 2.

Clusters 3 and 4 also revealed enrichment of certain biological processes, such as protein biosynthesis, protein metabolism and modification, and B cell immunity for cluster 3 and intracellular protein traffic, vesicle transport, and amino acid metabolism for cluster 4 .

\section{EGCG increases expression of genes related to protein biosynthesis and protein metabolism/ modification}

Treatment with EGCG led to elevated expression of genes associated with protein biosynthesis and protein modification (Table 1), including eukaryotic translation

Table 2. Intracellular protein traffic-related genes regulated by EGCG

\begin{tabular}{|c|c|c|c|c|c|}
\hline Probe id & Gene symbol & Gene name & $\begin{array}{c}6 h \text { ratio } \\
-\log 2\end{array}$ & $\begin{array}{c}12 \mathrm{~h} \text { ratio } \\
-\log 2\end{array}$ & $\begin{array}{c}24 \mathrm{~h} \text { ratio } \\
-\log 2\end{array}$ \\
\hline \multicolumn{6}{|c|}{ Intracellular protein traffic } \\
\hline 115761 & ARL1 & ADP-ribosylation factor-like 1 & 0.417 & 1.044 & 1.263 \\
\hline 217838 & $\mathrm{CHML}$ & choroideremia-like (Rab escort protein 2 ) & 1.105 & 1.738 & 2.416 \\
\hline 166994 & CHMP5 & chromatin modifying protein 5 & -0.001 & 0.433 & 0.887 \\
\hline 147300 & CSNK1G3 & casein kinase 1 , gamma 3 & -0.187 & 0.129 & 0.956 \\
\hline 171123 & EXOC2 & exocyst complex component 2 & 0.297 & -0.031 & 0.969 \\
\hline 159351 & KDELR2 & KDEL endoplasmic reticulum protein retention receptor 2 & 0.110 & 0.639 & 1.008 \\
\hline 157883 & NAPG & $\mathrm{N}$-ethylmaleimide-sensitive factor attachment protein, gamma & 0.781 & 1.421 & 2.015 \\
\hline 221608 & NUP107 & nucleoporin $107 \mathrm{kDa}$ & -0.159 & 1.404 & 1.182 \\
\hline 114948 & NUP50 & nucleoporin $50 \mathrm{kDa}$ & 0.663 & 1.031 & 1.202 \\
\hline 123733 & PPIL3 & peptidylprolyl isomerase (cyclophilin)-like 3 & 0.206 & 0.427 & 1.348 \\
\hline 156761 & RAB18 & RAB18, member RAS oncogene family & -0.068 & 0.419 & 0.736 \\
\hline 112058 & RAB8B & RAB8B, member RAS oncogene family & 0.980 & 1.329 & 1.549 \\
\hline 197182 & RER1 & RER1 retention in endoplasmic reticulum 1 homolog (S. cerevisiae) & -0.135 & 1.013 & 1.135 \\
\hline 155479 & RTN3 & reticulon 3 & 0.385 & 0.470 & 1.470 \\
\hline 181273 & SAR1A & SAR1 gene homolog A (S. cerevisiae) & 1.004 & 1.880 & 1.965 \\
\hline 171123 & SEC5L1 & SEC5-like 1 (S. cerevisiae) & 0.297 & -0.031 & 0.969 \\
\hline 169954 & SNX6 & sorting nexin 6 & 0.424 & 0.290 & 0.615 \\
\hline 209483 & TMED7 & transmembrane emp24 protein transport domain containing 7 & 0.779 & 1.297 & 1.787 \\
\hline 121895 & TOM1L1 & target of myb1-like 1 (chicken) & -0.043 & 0.758 & 1.459 \\
\hline 122318 & TRAM1 & translocation associated membrane protein 1 & -0.010 & 0.824 & 1.479 \\
\hline 209190 & WDR24 & WD repeat domain 24 & 0.254 & 2.244 & 4.139 \\
\hline 117439 & YIPF4 & Yip1 domain family, member 4 & 0.526 & 1.308 & 1.222 \\
\hline 163460 & YKT6 & SNARE protein Ykt6 & 0.859 & 1.436 & 1.625 \\
\hline
\end{tabular}


initiation factors (EIFs), mitochondrial ribosomal proteins (MRPLs), ribosomal proteins (RPLs), dual specificity phosphatases (DUSPs), peptidylprolyl isomerase $\mathrm{H}$ $(\mathrm{PPIH})$, protein phosphatase $2 \mathrm{~A}$, regulatory subunit B(PPP2R4), and protease, serine, 21(PRSS21). Many of these transcripts showed significant elevation in expression (more than 2-fold) at $12 \mathrm{~h}$.

\section{Effects of EGCG on intracellular protein traf- fic-related genes}

Many genes related to protein trafficking were upregulated by EGCG treatment (Table 2). Transcripts in this biological process showed a peculiar increase in expression at $24 \mathrm{~h}$. In particular, genes like choroideremia-like (CHML), N-ethylmaleimide-sensitive factor attachment protein gamma (NAPG), and WD repeat domain 24 (WDR24) showed overexpression, greater than 4-fold, at $24 \mathrm{~h}$.

\section{EGCG alters expression of several genes related to lipid metabolism}

EGCG treatment turned out to cause underexpression of many genes related to lipid metabolism (Table 3). The expression of genes involved in lipid transport, including caveolin 1 (CAV1), niemann-pick disease, type C1 (NPC1), oxysterol-binding protein-like 1A (OSBPL1A), and clusterin (CLU), were downregulated, as shown by PANTHER gene expression data analysis (Table 1). In particular, NPC1 has been reported to be involved in the intracellular trafficking of cholesterol (Pacheco and Lieberman, 2008). On the other hand, the expression of genes related to the cholesterol biosynthetic pathway, including isopentenyl-diphosphate delta isomerase 1 (IDI1), farnesyl-diphosphate farnesyltransferase 1 (FDFT1), 3-hydroxy-3-methylglutaryl-coenzymeA reductase (HMGCR), and HMG-coenzyme A synthase 1 (HMGCS1), was upregulated at $24 \mathrm{~h}$ after EGCG treatment. These observations indicate that treatment with EGCG induces the synthesis and accumulation of cholesterol in HepG2 cells.

Table 3. Lipid metabolism-related genes regulated by EGCG

\begin{tabular}{|c|c|c|c|c|c|}
\hline Probe id & Gene symbol & Gene name & $\begin{array}{c}6 h \text { ratio } \\
-\log 2\end{array}$ & $\begin{array}{c}12 \mathrm{~h} \text { ratio } \\
-\log 2\end{array}$ & $\begin{array}{c}24 \mathrm{~h} \text { ratio } \\
-\log 2\end{array}$ \\
\hline 185568 & PLA2G4A & phospholipase A2, group IVA (cytosolic, calcium-dependent) & -0.848 & -2.224 & -2.178 \\
\hline 158523 & PAFAH1B1 & platelet-activating factor acetylhydrolase, isoform $\mathrm{lb}$, alpha subunit $45 \mathrm{kDa}$ & -0.404 & -1.541 & -1.895 \\
\hline 128232 & PITPNM1 & phosphatidylinositol transfer protein, membrane-associated 1 & -0.454 & -0.656 & -1.644 \\
\hline 111955 & NR2F2 & nuclear receptor subfamily 2 , group $F$, member 2 & -0.540 & -0.758 & -1.513 \\
\hline 122343 & FABP5 & fatty acid binding protein 5 (psoriasis-associated) & -0.096 & -0.703 & -1.459 \\
\hline 114356 & ACSL3 & acyl-CoA synthetase long-chain family member 3 & -1.106 & -1.981 & -1.271 \\
\hline 137968 & OCRL & oculocerebrorenal syndrome of Lowe & 0.022 & -1.121 & -0.937 \\
\hline 102218 & ACLY & ATP citrate lyase & -0.452 & -1.418 & -0.794 \\
\hline 128174 & CLU & clusterin & -0.089 & -0.893 & -0.747 \\
\hline 128869 & TMEM23 & transmembrane protein 23 & -0.967 & -1.224 & -0.420 \\
\hline 171037 & ALDH3A2 & aldehyde dehydrogenase 3 family, member A2 & -0.119 & -1.163 & -0.414 \\
\hline 146593 & ACADVL & acyl-Coenzyme A dehydrogenase, very long chain & 0.733 & 0.820 & -0.036 \\
\hline 133876 & ACOT7 & acyl-CoA thioesterase 7 & 0.827 & 1.025 & 0.011 \\
\hline 199757 & CAV1 & caveolin 1 , caveolae protein, $22 \mathrm{kDa}$ & 0.353 & -0.485 & -1.061 \\
\hline 170041 & NPC1 & Niemann-Pick disease, type $\mathrm{C} 1$ & -0.052 & -0.583 & -0.446 \\
\hline 168754 & OSBPL1A & oxysterol binding protein-like $1 \mathrm{~A}$ & -0.642 & -0.998 & 0.255 \\
\hline 170102 & PMVK & phosphomevalonate kinase & 0.859 & 1.098 & 0.269 \\
\hline 119370 & PNPLA4 & patatin-like phospholipase domain containing 4 & 0.792 & 0.843 & 0.403 \\
\hline 112773 & PCYT1A & phosphate cytidylyltransferase 1 , choline, alpha & 0.528 & 0.959 & 1.090 \\
\hline 219614 & SAMD8 & sterile alpha motif domain containing 8 & 1.458 & 1.608 & 1.277 \\
\hline 126373 & STARD4 & START domain containing 4 , sterol regulated & -0.066 & -0.332 & 1.347 \\
\hline 145487 & GPAM & glycerol-3-phosphate acyltransferase, mitochondrial & 1.061 & 1.275 & 1.568 \\
\hline 231296 & PCSK9 & proprotein convertase subtilisin/kexin type 9 & 0.392 & 0.313 & 1.765 \\
\hline 161340 & PAFAH1B2 & platelet-activating factor acetylhydrolase, isoform $\mathrm{lb}$, beta subunit $30 \mathrm{kDa}$ & 1.380 & 2.608 & 1.990 \\
\hline 209566 & IDI1 & isopentenyl-diphosphate delta isomerase 1 & -0.698 & -0.951 & 1.651 \\
\hline 154827 & FDFT1 & farnesyl-diphosphate farnesyltransferase 1 & -0.129 & -0.266 & 1.399 \\
\hline 174731 & HMGCR & 3-hydroxy-3-methylglutaryl-CoenzymeA reductase & -0.248 & 0.201 & 2.073 \\
\hline 193122 & HMGCS1 & 3-hydroxy-3-methylglutaryl-Coenzyme A synthase 1 (soluble) & -0.082 & 1.00 & 2.820 \\
\hline
\end{tabular}


Table 4. Stress-related genes regulated by EGCG

\begin{tabular}{|c|c|c|c|c|c|}
\hline Probe id & Gene symbol & Gene name & $\begin{array}{c}\text { 6h ratio } \\
-\log 2\end{array}$ & $\begin{array}{c}12 \mathrm{~h} \text { ratio } \\
-\log 2\end{array}$ & $\begin{array}{c}24 h \text { ratio } \\
-\log 2\end{array}$ \\
\hline \multicolumn{6}{|c|}{ Transcription factors } \\
\hline 185687 & ATF3 & activating transcription factor 3 & 2.645 & 4.101 & 2.504 \\
\hline 183867 & ATF4 & activating transcription factor 4 & 0.801 & 0.959 & 0.835 \\
\hline 100596 & CEBPG & CCAAT/enhancer binding protein (C/EBP), gamma & 1.603 & 2.299 & 1.792 \\
\hline 194114 & FOXO3A & forkhead box $\mathrm{O} 3 \mathrm{~A}$ & 1.092 & 1.695 & 1.038 \\
\hline \multicolumn{6}{|c|}{ Cell redox homeostasis/oxidative stress } \\
\hline 140616 & DUSP23 & dual specificity phosphatase 23 & 0.982 & 1.245 & 0.550 \\
\hline 121612 & DUSP5 & dual specificity phosphatase 5 & 2.526 & 1.996 & 0.794 \\
\hline 161352 & DUSP3 & dual specificity phosphatase 3 (vaccinia virus phosphatase VH1-related) & 0.244 & 1.611 & 1.166 \\
\hline 196828 & GCLC & glutamate-cysteine ligase, catalytic subunit & 0.388 & 0.370 & 1.032 \\
\hline 196828 & PRDX5 & peroxiredoxin 5 & 0.494 & 0.728 & 1.034 \\
\hline 149133 & SOD2 & Superoxide dismutase 2 , mitochondrial & 0.939 & 1.383 & 1.315 \\
\hline 226167 & TXNDC10 & thioredoxin domain containing 10 & 0.541 & 1.117 & 1.528 \\
\hline 179796 & TXN2 & thioredoxin 2 & 0.125 & 1.274 & 0.720 \\
\hline 197013 & SDF2L1 & stromal cell-derived factor 2-like 1 & 1.098 & 1.694 & 0.846 \\
\hline \multicolumn{6}{|c|}{ DNA damage stimulus } \\
\hline 144049 & BTG1 & B-cell translocation gene 1 , anti-proliferative & 0.717 & 1.875 & 2.370 \\
\hline 192182 & RAD1 & RAD1 homolog (S. pombe) & -0.109 & 0.568 & 1.232 \\
\hline 234387 & RAD23B & RAD23 homolog B (S. cerevisiae) & -0.296 & -1.267 & -1.587 \\
\hline 184090 & RAD18 & RAD18 homolog (S. cerevisiae) & -0.044 & -0.644 & 0.319 \\
\hline \multicolumn{6}{|c|}{ Cell cycle/apoptosis } \\
\hline 179446 & BNIP2 & BCL2/adenovirus E1B 19kDa interacting protein 2 & 0.617 & 1.222 & 1.106 \\
\hline 109165 & BNIP3 & BCL2/adenovirus E1B 19kDa interacting protein 3 & 0.205 & 1.045 & 2.112 \\
\hline 144168 & BNIP3L & BCL2/adenovirus E1B $19 \mathrm{kDa}$ interacting protein 3-like & 0.900 & 1.498 & 2.152 \\
\hline 114136 & CASP7 & caspase 7, apoptosis-related cysteine peptidase & 0.286 & 0.453 & 0.806 \\
\hline 235779 & CDK6 & cyclin-dependent kinase 6 & -0.698 & -1.874 & -2.044 \\
\hline 207914 & CCNJ & cyclin J & -0.685 & -1.671 & -1.456 \\
\hline 207292 & CCNT2 & cyclin T2 & -1.274 & -1.851 & -1.330 \\
\hline 204006 & CDKN2B & cyclin-dependent kinase inhibitor 2B ( $\mathrm{p} 15$, inhibits CDK4) & 1.391 & 1.147 & 0.910 \\
\hline 137292 & CDKN1A & cyclin-dependent kinase inhibitor 1A (p21, Cip1) & 1.707 & 2.005 & 1.254 \\
\hline 175225 & NFKBIE & nuclear factor of kappa light polypeptide gene enhancer in B-cells inhibitor, epsilon & 1.402 & 1.167 & 0.238 \\
\hline 107316 & PTEN & phosphatase and tensin homolog & -0.120 & -0.184 & 0.898 \\
\hline
\end{tabular}

\section{EGCG increases expression of cellular stress and ER stress markers}

Treatment with EGCG resulted in elevated expression of cellular stress markers, such as transcription factors, markers of DNA damage, cell cycle/apoptosis, and genes associated with redox status (Table 4). Of particular note, 3 genes-activating transcription factor 3 (ATF3), activating transcription factor 4 (ATF4), and CCAAT/enhancer binding protein (C/EBP), gamma (CEBPG)-are known to be associated with ER stress. The involvement of ER stress in EGCG-induced cytotoxicity was further monitored by real-time RT-PCR of differentially expressed marker genes (Fig. 3). The results showed that ATF3, which is a member of the ATF/CREB subfamily of the basic region leucine zipper (bZIP) family, was increased by more than 8-fold in 6-h-treated cells. In addition, members of the CCAAT/enhancer binding protein (C/EBP) family of transcription factors, such as the apoptosis-inducing proteins GADD153 (CHOP) and GADD34, were also upregulated in 6-h-treated cells. The expression of GADD153 and GADD34 was increased more than 2-fold at all time points.

Prolonged ER stress can perturb mitochondria, triggering oxidative stress. $\mathrm{Ca}^{2+}$-induced oxidative stress can cause both cell death and activation of NF- $\mathrm{K} B$ signaling (Ji and Kaplowitz, 2006). In this study, NFKBIE was differentially expressed at $6 \mathrm{~h}(2.6$-fold) and $12 \mathrm{~h}$ (2.2-fold), but at $24 \mathrm{~h}$ it was not significantly expressed (1.1-fold). NFKBIE is known as a member of the family of NF- $\kappa \mathrm{B}$ inhibitory proteins (l $\kappa \mathrm{Bs})$ that interact with $\mathrm{NF}-\kappa \mathrm{B}$ in unstimulated cells (Komissarova et al., 2008),

\section{Verification of microarray data}

To verify the gene expression profiles from the oligonucleotide microarray, 6 genes that showed significant up- 

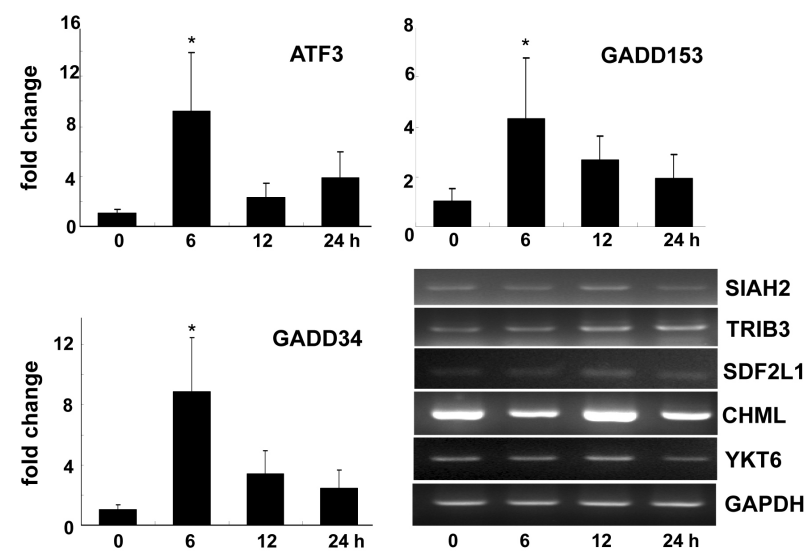

Fig. 3. Time sequences of gene expression, assayed by real-time and semiquantitative RT-PCR. Real-time PCR analysis was performed for GADD153, GADD43, and ATF3. Each value is expressed as the fold-change in mRNA levels at each time point and represents the mean \pm SEM of 3 independent experiments. Semiquantitative RT-PCR was also performed to verify the relative mRNA levels of randomly selected genes.

regulation were selected and subjected to semiquantitative RT-PCR analysis. The expression pattern observed by PCR showed a concordance with the pattern assayed by microarray (Fig. 3).

\section{Discussion}

The objective of this study was to determine the effects of EGCG on hepatic gene expression and to understand the underlying mechanism of hepatotoxicity for this polyphenol in vitro. Our study revealed that high concentrations of EGCG affected the expression of a large number of genes in various cellular pathways (1343 genes). The functional enrichment analysis of the differentially expressed genes provided new insight into the molecular mechanism of EGCG for its undesirable effect.

We showed that high concentrations of EGCG activate the unfolded protein response (UPR), resulting in increased expression of ER stress response genes in cultured human HepG2 cells. ER not only is the place of protein synthesis and package; it also plays a central role in various signaling pathways. Dysfunction of the ER can be induced by various biological disturbances, so-called ER stress. UPR is one of the well-known ER stresses, and it may be associated with cellular apoptosis in HepG2 cells. The UPR is reported to be required for safe-guarding protein synthesis, post-translational modifications, folding and secretion, calcium storage, and calcium signaling in the normal physiological state.

In our study, EGCG exposure to HepG2 cell cultures resulted in a marked increase in the mRNA level of genes associated with protein synthesis and modification. This is not surprising, because repairing hepatic damage requires the massive production of new proteins and modification of proteins. EGCG treatment also revealed upregulation of many genes involved in protein secretion, including intracellular protein trafficking. These expression patterns were consistent with those of pyrazole, a known inducer of ER stress (Nichols and Kirby, 2008).

EGCG-induced upregulation of GADD34, GADD153, and ATF3 provides genetic evidence for the presence of EGCG-caused ER stress. Even though GADD153, a member of the C/EBP gene family of transcription factors, has already been reported to be induced by growth arrest, DNA damage, or UV irradiation, recent studies have demonstrated that the gene also has great responsivity to ER stress (Outinen et al., 1999; Wang et al., 1996). In addition, it has previously been shown that GADD153 induces cell death by promoting protein synthesis and oxidation in the stressed endoplasmic reticulum (Marciniak et al., 2004). The transcription of ATF3 usually increases within $2 \mathrm{~h}$ from stress exposure, and ATF3 protein can function as a homodimer or as a member of the complex of the CCAAT/enhancer binding protein (C/EBP) family of transcription factor, such as GADD153. Jiang et al. (2003) also found that ATF3 is induced in response to ER stress. GADD34 encodes a regulatory subunit of an elF2 $\alpha$-directed-phosphatase complex that promotes the recovery of protein synthesis in cells exposed to ER stress, causing more ER stress (Lai et al., 2007).

In addition to genes related to ER stress, apoptosis-related genes, like BNIP3 and BNIP3L, were also upregulated around 2-fold after $12 \mathrm{~h}$ and around 4-fold after $24 \mathrm{~h}$ of EGCG treatment in HepG2 cells. According to a previous report, induction of BNIP3 localized itself to mitochondria and triggered a loss of membrane potential and an increase in reactive oxygen species production, which often lead to cell death (Burton and Gibson, 2009). These results imply that EGCG exposure to HepG2 cells induces BNIP-mediated apoptosis through disturbances of ER functions.

There have been various studies to elucidate the molecular mechanism of EGCG activity. Although some EGCG-interacting molecules have been identified, the molecular mechanism of its cytotoxic activity is not yet fully clarified. Recently, Kuzuhara et al. (2006) reported that EGCG and its related derivatives that have a galloyl group interacted with DNA and RNA directly, while catechin and its related derivatives, lacking a galloyl group, 
did not interact with DNA or RNA. On the other hand, Ermakova et al. (2006) reported that glucose-regulating protein 78 (GRP78), a molecular chaperone in the ER, is an EGCG-binding protein. In the report, EGCG and catechins, containing a galloyl group, showed stronger inhibition of GRP78 than EC, EGC, and catechins that lacked a galloyl group. This observation strongly supports the existence of an interaction between the galloyl group and GRP78.

ER stress contributes to intracellular lipid accumulation, which is mediated by ER-associated transmembrane sterol response element-binding protein 1 (SREBP1), an ER membrane-bound transcription factor. SREBP1 activates genes encoding enzymes in the cholesterol/triglyceride biosynthesis and uptake pathway ( $\mathrm{Ji}$ and Kaplowitz, 2006). The present study showed that EGCG-induced ER stress increased the expression of genes responsible for cholesterol/triglyceride biosynthesis, including HMGCR and HMGCS1, and decreased the expression of genes responsible for lipid transport, including CAV1, NPC1, OSBPL1A, and CLU. Interestingly, clusterin (CLU), a multifunctional heterodimeric glycoprotein, is known for its implication in a wide range of physiological functions, such as lipid transport, tissue repair and remodeling, membrane protection, and promotion of cell interaction. It has been reported that induction of clusterin may represent a protective response against oxidative stress (Shannan et al., 2006). More recently, clusterin has been proposed as a candidate biomarker for kidney injury (Sieber et al., 2009).

Based on the analyses of the presented microarray data, it seems clear that high concentrations of EGCG cause cell death, probably via the apoptosis pathway, by inducing ER stress and the expression of ER stress response genes (GADD153, GADD34, and ATF3). The presented results strongly suggest that high concentrations of EGCG might cause fatty liver through ER stress-induced accumulation of lipids in living subjects. The identification of other regulatory genes may also aid in clarifying the molecular mechanism underlying EGCG-induced hepatotoxicity.

\section{References}

Burton, T.R., and Gibson, S.B. (2009). The role of Bcl-2 family member BNIP3 in cell death and disease: NIPping at the heels of cell death. Cell Death Differ. 16, 515-523.

Dodo, K., Minato, T., Noguchi-Yachide, T., Suganuma, M., and Hashimoto, Y. (2008). Antiproliferative and apoptosis-inducing activities of alkyl gallate and gallamide derivatives related to (-)-epigallocatechin gallate. Bioorg. Med. Chem. 16, 7975-7982.

Ermakova, S.P., Kang, B.S., Choi, B.Y., Choi, H.S., Schuster, T.F., Ma, W.Y., Bode, A.M., and Dong, $Z$.
(2006). (-)-Epigallocatechin gallate overcomes resistance to etoposide-induced cell death by targeting the molecular chaperone glucose-regulated protein 78. Cancer Res. 66, 9260-9269.

Galati, G., Lin, A., Sultan, A.M., and O'Brien, P.J. (2006). Cellular and in vivo hepatotoxicity caused by green tea phenolic acids and catechins. Free Radic. Biol. Med. 40, 570-580.

Goodin, M.G., and Rosengren, R.J. (2003). Epigallocatechin gallate modulates CYP450 isoforms in the female Swiss-Webster mouse. Toxicol. Sci. 76, 262-270.

Isbrucker R.A., Edwards, J.A., Wolz, E., Davidovich, A., and Bausch, J. (2006). Safety studies on epigallocatechin gallate (EGCG) preparations. Part 3: teratogenicity and reproductive toxicity studies in rats. Food Chem. Toxicol. 44, 651-661.

Ji, C., and Kaplowitz, N. (2006). ER stress: can the liver cope? J. Hepatol. 45, 321-333.

Jiang, H.Y., Wek, S.A., McGrath, B.C., Lu, D., Hai, T., Harding, H.P., Wang, X., Ron, D., Cavener, D.R., and Wek, R.C. (2004). Activating transcription factor 3 is integral to the eukaryotic initiation factor 2 kinase stress response. Mol. Cell. Biol. 24, 1365-1377.

Komissarova, E.V., Li, P., Uddin, A.N., Chen, X., Nadas, A., and Rossman, T.G. (2008). Gene expression levels in normal human lymphoblasts with variable sensitivities to arsenite: identification of GGT1 and NFKBIE expression levels as possible biomarkers of susceptibility. Toxicol. Appl. Pharmacol. 226, 199-205.

Kuzuhara, T., Sei, Y., Yamaguchi, K., Suganuma, M., and Fujiki, H. (2006). DNA and RNA as new binding targets of green tea catechins. J. Biol. Chem. 281, 17446-17456.

Lai, E., Teodoro, T., and Volchuk, A. (2007). Endoplasmic reticulum stress: signaling the unfolded protein response. Physiology 22, 193-201.

Lambert, J.D., Sang, S., and Yang, C.S. (2007). Possible controversy over dietary polyphenols: benefits vs risks. Chem. Res. Toxicol. 20, 583-585.

Marciniak, S.J., Yun, C.Y., Oyadomari, S., Novoa, I., Zhang, Y., Jungreis, R., Nagata, K., Harding, H.P., and Ron, D. (2004). CHOP induces death by promoting protein synthesis and oxidation in the stressed endoplasmic reticulum. Genes Dev. 18, 3066-3077.

Nichols, K.D., and Kirby, G.M. (2008). Microarray analysis of hepatic gene expression in pyrazole-mediated hepatotoxicity: identification of potential stimuli of Cyp2a5 induction. Biochem. Pharmacol. 75, 538-551.

Outinen, P.A., Sood, S.K., Pfeifer, S.I., Pamidi, S., Podor, T.J., Li, J., Weitz, J.I., and Austin, R.C. (1999). Homocysteine-induced endoplasmic reticulum stress and growth arrest leads to specific changes in gene expression in human vascular endothelial cells. Blood 94, 959-967.

Pacheco, C.D., and Lieberman, A.P. (2008). The pathogenesis of Niemann-Pick type $C$ disease: a role for autophagy? Expert Rev. Mol. Med. 10, e26.

Schmidt, M., Schmitz, H.J., Baumgart, A., Guédon, D., Netsch, M.I., Kreuter, M.H., Schmidlin, C.B., and Schrenk, D. (2005). Toxicity of green tea extracts and 
106 Genomics \& Informatics Vol. 7(2) 97-106, June 2009

their constituents in rat hepatocytes in primary culture. Food Chem. Toxicol. 43, 307-314.

Shannan, B., Seifert, M., Leskov, K., Willis, J., Boothman, D., Tilgen, W., and Reichrath, J. (2006). Challenge and promise: roles for clusterin in pathogenesis, progression and therapy of cancer. Cell Death Differ. 13, 12-19.

Sieber, M., Hoffmann, D., Adler, M., Vaidya, V.S., Clement, M., Bonventre, J.V., Zidek, N., Rached, E., Amberg, A., Callanan, J.J., Dekant, W., and Mally, A. (2009). Comparative analysis of novel noninvasive renal biomarkers and metabonomic changes in a rat model of gentamicin nephrotoxicity. Toxicol. Sci. 109, 336-349.
Vittal, R., Selvanayagam, Z.E., Sun, Y., Hong, J., Liu, F., Chin, K.V., and Yang, C.S. (2004). Gene expression changes induced by green tea polyphenol (-)-epigallocatechin-3-gallate in human bronchial epithelial 21BES cells analyzed by DNA microarray. Mol. Cancer Ther. 3, 1091-1099.

Wang, X.Z., Lawson, B., Brewer, J.W., Zinszner, H., Sanjay, A., Mi, L.J., Boorstein, R., Kreibich, G., Hendershot, L.M., and Ron, D. (1996). Signals from the stressed endoplasmic reticulum induce $\mathrm{C} / \mathrm{EBP}$-homologous protein (CHOP/GADD153). Mol. Cell. Biol. 16, 4273-4280. 